\title{
Exploiting Link Structure for Web Page Genre Identification
}

\author{
Jia Zhu • Qing Xie • Shoou-I Yu
}

Received: date / Accepted: date

\begin{abstract}
As the World Wide Web grows at an unprecedented pace, web page genre identification has recently attracted increasing attention because of its importance in web search. A common approach for genre identification is to utilize textual features that can be extracted directly from the web page itself, i.e., On-Page features. The extracted features are subsequently given to a machine learning algorithm that will perform classification. However, these approaches may not be effective when the web page contains limited textual information (e.g., full of images). In this paper, we tackle the genre identification of web pages in such situation. We propose a framework that not only uses On-Page features, but also takes into account information in neighboring pages, i.e., the pages that are connected to the original page by backward and forward links. We first introduce a graph-based model called GenreSim which selects an appropriate set of neighboring pages. We then construct a multiple classifier combination module that utilizes information from the selected neighboring pages and On-Page features to improve genre identification performance. The experiments are conducted on well-known corpora, and the favorable results indicate that our proposed framework is effective, particularly in identifying web pages with limited textual information.
\end{abstract}

Keywords Genre Identification · Neighboring Pages Selection · Multiple Classifiers

J. Zhu

School of Computer Science, South China Normal University, China

E-mail: jzhu@m.scnu.edu.cn

Q. Xie

Division of Computer, Electrical and Mathematical Sciences and Engineering, King Abdullah University of Science and Technology, Saudi Arabia

S. $\mathrm{Yu}$

School of Computer Science, Carnegie Mellon University, USA 


\section{Introduction}

As the World Wide Web grows at an unprecedented pace, the importance of web page categorization to enhance web search has increased substantially. Web page categorization can be divided into two main sub-problems: topic categorization and genre categorization, and our work will discuss the problems of genre categorization (also known as genre identification). Different from the topic categorization that identifies the information contained in the detailed contents of the web pages [39], genre categorization focuses more on the functional purposes of the web page and discovers groups of texts that share a general form of transmission, purpose, or discourse properties. By this means, genre can be defined as a term for the category or form of a set of web pages. For example, a website like www.news.com.au, has many pages in different topics, e.g., sport news and finance news, but the genre of these pages in this website is news. Genre identification has recently attracted increasing attention because it can be used to significantly improve the quality of web and document search results $[1,4,8,31,37]$. With genre identification, a search engine can label the genre of each retrieved result for the users to save their time, as exemplified in Fig. 1 using WEGA Firefox plugin ${ }^{1}$.

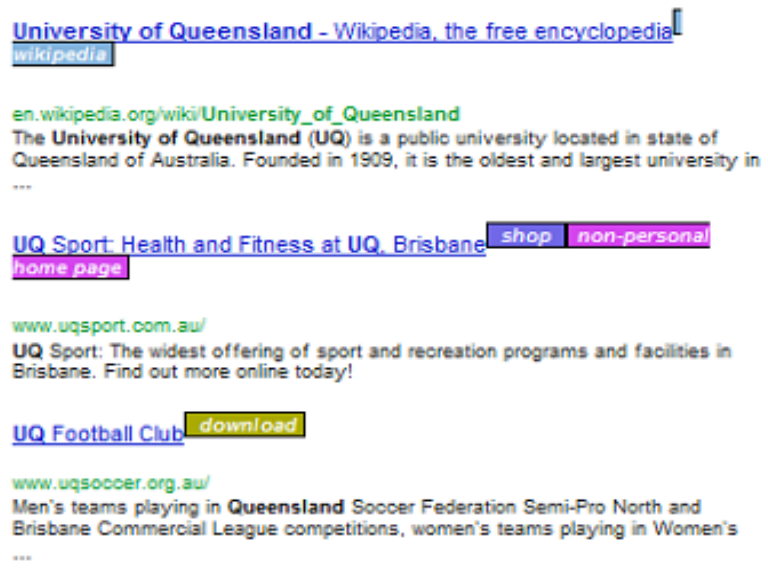

Fig. 1: Sample of labeled search results

The majority of existing approaches of genre identification are limited to utilizing information on the page, namely, On-Page features. On-Page features include textual information, e.g., bag-of-words(BOW) and term frequency [15, 38], and structural information [5,8], e.g., HTML tags. Textual information represents the web page content while structural information specifies how the content is presented. However, in a particular web page, these features

1 http://www.uni-weimar.de/en/media/chairs/webis/ research/projects/wega/ 
are sometimes missing or unrecognizable for various reasons. For example, as shown in Fig. 2, some web pages contain a large number of images or videos and very few textual content. In this case, it is difficult for a classifier that depends solely on On-Page textual features to correctly identify the genre of the web page.

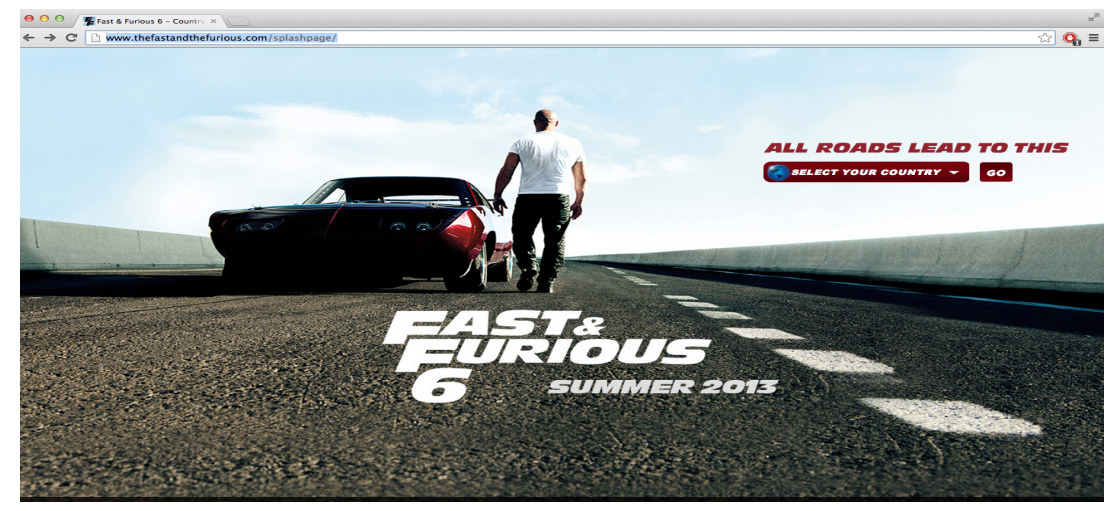

Fig. 2: A web page with few textual information

In light of the aforementioned limitations, we propose a framework that utilizes additional information from the neighboring pages of a given web page to enhance genre identification. Neighboring pages are web pages that are connected to a given web page by either forward or backward links. The main motivation for utilizing the additional information from these pages is that, they usually contain pages of similar genre as the original page and thus contain discriminative textual information for recognizing the genre of the given web page. Thus, utilizing the linkage information to harvest additional textual features could provide more references for classifiers to learn a better classification model $[6,27,32]$. One problem with using information from neighboring pages is that one page may have numerous neighboring pages which are likely to belong to different web genres. However, we are only interested in those neighboring pages that are in the same genre as the original page. Therefore, we propose a link-based graph model called GenreSim which exploits a link structure to select relevant neighboring pages. Compared to existing method$\mathrm{s}$, our model takes into account as many neighboring pages as possible while greatly reducing the noisy information by only selecting an appropriate subset of relevant neighboring pages. In addition, to further improve the classification performance, we construct a multiple classifier combination module to combine the outputs from various classifiers that use different features. The proposed framework is evaluated using benchmark corpora for web page genre identification, and the favorable results indicate the effectiveness of our method.

In sum, our main contributions are threefold compared to a previous work [42]: 
1. We propose a link-based graph model called GenreSim, which exploits link structure to select relevant neighboring pages. The selected neighboring web pages are utilized to enhance genre identification of the original web page. We improve the selection by considering the probability for a page to have a given number of backward links.

2. We propose a multiple classifier combination model that combines outputs of multiple classifiers to improve genre identification.

3. Extensive experiments with statistical tests on standard benchmark corpora demonstrate the effectiveness of our method.

The rest of this paper is organized as follows. In Section 2, we discuss related works in web page genre identification. In Section 3, we describe the main challenges of genre identification and the details of our framework. In Section 4, we present our experiments, evaluation metrics, and results. We conclude this study in Section 5 .

\section{Related Work}

Previous studies on web page genre identification differ significantly with respect to two factors: 1) the feature set used to represent the content and structure of web pages and 2) the method used to classify web pages based on the chosen feature set. The following review of some previous important works is presented in chronological order.

Meyer and Stein [8] provided a corpus of eight genres following a user study on genre usefulness with a support vector machine classifier. They also examined various feature sets in an attempt to combine different kinds of information including, word frequencies, text statistics, and parts of speech frequencies. All these information are textual information that will also be used in our framework.

Kennedy and Shepherd [14] focused on a specific genre and its sub-genres. Using a neural network, they attempted to discriminate between home pages and non-home pages. Their feature set comprises features about the content (e.g., common words and meta tags), form (e.g., number of images), and functionality (e.g., number of links and use of scripts). The best reported results were for personal home pages.

Finn and Kushmerick [9] investigated different feature sets for genre classification, including bag-of-words (BOW), parts of speech frequencies, and text statistics. They studied how these features can be combined to maximize accuracy both on a single topic and across topics. Their model was generated by C4.5, and they evaluated how the classifier can perform well either in a single domain or for domain transfer with ensemble and active learning.

Santini [34] studied web page genre classification based on three different feature sets, including frequencies of common words, parts of speeches and parts of speech trigrams, HTML tags, punctuation marks, and so forth She built a corpus and evaluated her approach to predict the genre of unclassified web pages with support vector machine and naive bayes classifiers. However, 
the author could not fully evaluate the model because of the lack of genre benchmarks. Later, the author further analyzed web page genre classification in terms of genre hybridism and individualization [35]. Genre hybridism accounts for genres variation within web pages, while individualization refers to the absence of any recognized genre in a web page. Therefore, the author claimed that web pages need a zero-to-multi-genre classification scheme in addition to the traditional single-genre classification. We applied this concept into our approach, but select the genre with the highest score to a page rather than mark a page with multiple genres.

Kanaris and Stamatatos [13] proposed low-level feature sets of variablelength character n-grams and combined this representation with information about the most frequent HTML tags. Based on two benchmark corpora, they showed that support vector machine (SVM) based classification approach can improve the results in both cases compared with other machine learning approaches. Based on their research outcome, we selected SVM to be the classification method in our proposed framework.

Dong et al. [7] examined the effect of various attributes on four different web genres, namely, FAQ, News, E-Shopping, and Personal Home Pages, by using the Naive Bayes classifier and the Information Gain Measure for feature selection. The results indicated that fewer features produce better precision, but more features produce better recall and combined attributes will always perform better than single attributes.

Chen and Choi [6] defined five top-level genre categories and developed new methods to extract 31 features from web pages, which analyzed not only the contents, but also other features, such as HTML tags and Java scripts. Their evaluation results showed that additional features can help the classifier to better learn the classification model.

Jebari [11] proposed a new centroid-based approach for web pages genre categorization using a set of genre-labeled web pages. The obtained centroids from these labeled pages are used to classify new web pages. Each web page is assigned to all predefined genres with confidence scores, and the centroids are refined after classification. The feature sets include URL addresses, logical structures and hypertext structures. The experiments conducted on two known corpora show that his approach is very fast and outperforms other machine learning approaches that require data training.

Mason et al. [26] further extended previous works that use n-gram representation of a web page to automatically classify web pages by genre as these n-gram based methods have high-precision lower-recall rates. Their experiments showed their approach can assign more labels than previous works while retaining high accuracy. However, similar to most of the existing research, we only focus on the accuracy of the single-label classification in this article, which means each web page only belongs to one genre.

Sharoff et al. [37] investigated the performance of several types of features and found that one of the n-grams features performs best on their experiments, but its performance may not be transferable to the wider web due to the lack of comparability between different annotation labels. Although their experiments 
are comprehensive, their finding is limited and will not work when the textual information in the page is not sufficient, which is one of the reasons we do not use the full text in our approach.

Kim and Ross [16] reported that term distribution pattern is a better indicator to determine genre class than term frequency. Their approach is not intended for web pages, but some of their research outcomes, e.g., use the derived document structural information from a scientific article and locate target information to more accurately extract text information, can also be references in web page genre identification.

Kumari and Reddy [19] recently proposed an approach called Combined Stemming Approach. They focused on extracting genre-relevant words based on word level and linguistic features in order to improve the classification accuracy with techniques of combined stemming and stop-word elimination. Their experimental results showed the superior performance of their proposed method on the test dataset.

All the above approaches we discussed focused only on On-Page information, which may not be effective when there is only sparse textual information on the pages. Abramson and Aha [1] presented a method that used information from URLs for web page genre classification because some URLs may contain text that indicates the genre, e.g., blog. This approach can partially solve the problem, but it is still not a general approach for all websites. For example, facebook.com and twitter.com are both social network websites, yet it is difficult to determine if they should belong to the same genre from their URLs.

Pritsos and Stamatatos [31] focused on the use of content information with different text representation methods. In addition, they examined base SVM learners and an ensemble of classifiers. The results demonstrated that their approach can achieve very high precision and still maintain relatively high recall. However, their approach still cannot work well when there is limited information in web pages.

Compared to the existing work discussed above, our proposed framework not only uses textual and structural information, but also information from selected relevant neighboring pages. We further improve the classification accuracy by using combinations of multiple classifiers. The details of the framework are introduced in the next section.

\section{Proposed Framework}

Our proposed framework identifies web page genre based on both On-Page features and features from relevant neighboring pages. The features are selected by the feature selection module and used to train multiple classifiers. In the testing phase, the prediction scores acquired from multiple classifiers are given to the multiple classifiers combination module to obtain a single prediction score. The proposed framework is shown in Fig. 3. 


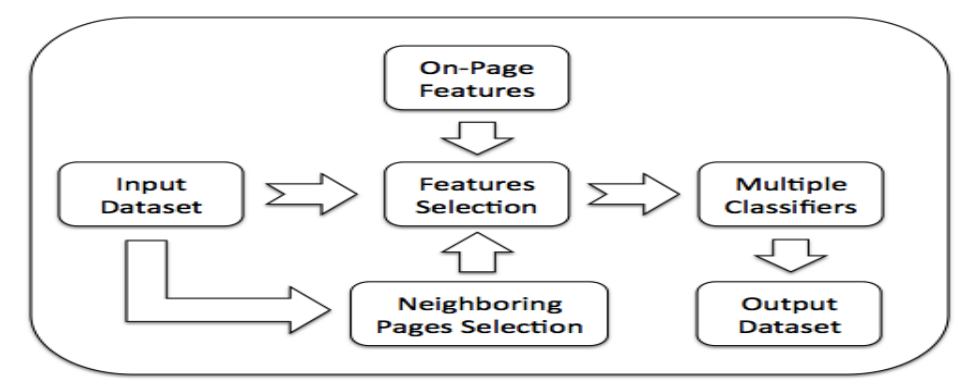

Fig. 3: Proposed Framework

\subsection{On-Page Features}

On-Page features capture the textual and structural information in a given web page. Structural features mainly capture the layout of the web page, whereas textual features represent the content of the web page. The textual information used in our method are page URL, title, keywords, headings, and anchors. Similar to many of the existing works discussed in Section 2, we do not use the text in between < body $>$ tags because the types of information we require are a straightforward and rich resource for a classifier to perform genre identification compared to the text in the body. For example, if the URL contains some specific words like "faq," "cv," and "how," the web genre can be easily recognized as these words provide very clear information. Moreover, for those web pages with limited textual information, these words are the most easily accessible source; thus, we select them as the textual feature. We apply Term Frequency-Inverse Document Frequency (TF-IDF) to represent all the text we extracted as a vector. TF-IDF is one of the most popular statistics in text mining. The calculated term weighting and inverse document frequency are used to score and rank a document's relevance [33]. The TF-IDF score for each term is calculated as follows:

$$
\text { TermScore }_{i j}=\frac{n_{i j}}{\sum_{k} n_{k j}} \times \log \frac{|D|}{\left|\left\{d: t_{i} \in d\right\}\right|}
$$

where $n_{i j}$ is the number of occurrences of the term $t_{i}$ in document $d_{j}$, the denominator is the sum of the occurrences of all terms in document $d_{j},|D|$ is the total number of documents in the corpus, and $\left|\left\{d: t_{i} \in d\right\}\right|$ is number of documents where the term $t_{i}$ appears. In our case, each document is a web page. Eventually, we have a vector that is a list of TF-IDF-represented terms we extracted from each web page.

Structural features are utilized by our method through computing the frequency of different HTML tags. Following [7,8,23], the HTML tags for which frequency is computed are images, links, emails, forms, tables, and div tags. However, according to existing works and our preliminary evaluations, structural information used independently cannot get sound results, especially if the 
dataset contains various genres of web pages. Therefore, we combine structural information with textual information to form the On-Page features.

\subsection{Neighboring Pages Selection Module}

On-Page features are not sufficient for web pages genre identification if the page has limited text information. Therefore, we propose to retrieve additional information from neighboring pages. The additional information can provide further evidence for classifiers to perform more reliable predictions. It is highly likely that some neighboring pages do not belong to the same genre as the original page. Therefore, selecting only the relevant neighboring pages from the large pool of neighboring pages is crucial. In the next few paragraphs, we will first detail how the neighboring page set is created for each web page, and then we will describe how our link-based similarity measure GenreSim selects the appropriate neighboring pages.

Neighboring pages are web pages connected to the original web page by forward or backward links [32]. Specifically, level-1 neighboring pages are the web pages immediately connected to a given web page by either forward or backward links. Level-2 neighboring pages are web pages that are two hyperlinks away from the given web page. For example, in Fig. $4, P$ is the original page. $B, D$, and $A, C, E, F$ are the level-1 and level-2 neighboring pages of $P$ respectively. In our model, the radius we consider is up to two hops because it is the farthest hop to the original page from the backward and forward links of level-1 neighboring pages. Taking further pages into account, e.g., three hops, will not only hugely increase the amount of computation, but will also receive more noisy information that will decrease the classification performance according to our past observation and experiments. For instance, assume a page has 10 neighboring pages in average, and $50 \%$ of the neighboring pages are not the same genre as the original page. This difference means we need to filter out $10^{*} 10^{*} 10^{*} 0.5=500$ useless pages in the case if we consider three hops.

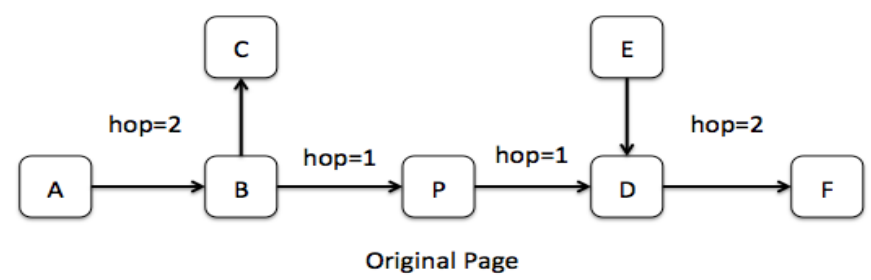

Fig. 4: Neighboring Pages of Page $\mathrm{P}$ 
We propose a link-based similarity measure called GenreSim, which is derived from the PageSim similarity measure [24], to select relevant neighboring web pages. Compared to other popular link-based similarity measures like SimRank[12] and Co-citation[33], PageSim can measure the similarity between any two pages without the need for intermediate pages as required in SimRank. PageSim can also consider neighboring pages in multiple hops in contrast to Co-citation which only considers immediate neighboring pages.

We model all the neighboring pages and the original page as a directed graph $G=(V, E)$ with vertices $V$ representing web pages $v_{i}(i=0,1,2, \ldots, n)$ and directed edges $E$ representing hyperlinks among the web pages. Page $v_{0}$ is the original page. Below is a list of definitions for our model.

Definition 1 Path: $p(u, v)$ is a path denoting a sequence of vertices from vertex $u$ to vertex $v$ through a set of edges $e_{i}(i=1,2, \ldots, m), e_{i} \in E$, and $E \in G$.

Definition 2 Path Set: PATH $(u, v)$ is the set of all possible paths from vertex $u$ to $v$.

Definition 3 Backward and Forward Links: $B(v)$ is the set of vertices representing the neighboring pages with a backward link to vertex $v$, and $F(v)$ is the set of vertices representing the neighboring pages with a forward link to vertex $v$.

Definition 4 Page Score: Score (v) is the query-independent score of a page $v$, which represents the importance of $v$ in the web graph according to its backward and forward links.

The original PageSim similarity measure only uses forward links, while in GenreSim, we use both forward and backward links because the number of backward links of a page can give us a general estimate of its prominence on the web graph [17]. In addition, we implement a weighted method to compute the page score and make it suitable for genre identification. A hyperlink from a page $u$ to $v$ can be considered as the recommendation of page $v$ by $u$, and thus $u$ and $v$ should have some kind of similarity [2]. Naturally, the recommendation decreases along with the links. We then define the recommendation score $\operatorname{Score}(u, v)$ of page $u$ propagating to $v$ through $P A T H(u, v)$ as shown in Equation (2):

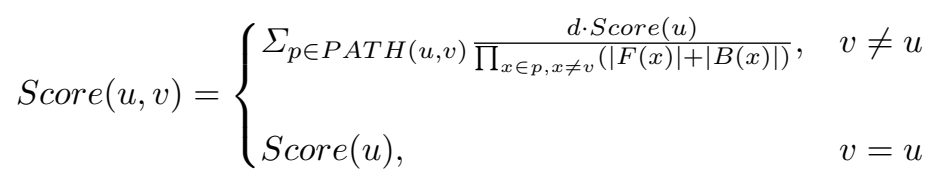

where $d \in(0,1]$ is a decay factor that is constant to make the score decrease along with the path. As we do not concentrate on optimizing $d$ in this work, we simply set the value of the decay factor to 0.5 , which is a common setting used by many graph based approaches. It means the score is decreased by half for each hop in the path. $\operatorname{Score}(u)$ is the page score for page $u$ and $u, v \in V$. 
To compute $\operatorname{Score}(u)$ in Equation (2), we extend the Hypertext-induced topic selection [18] algorithm to calculate the score.

In the web page graph $G$ we defined above, web pages that have many links pointing to them are called authorities, while web pages that have many outgoing links are called hubs. Better authorities are given by incoming edges from good hubs and better hubs come from outgoing edges to good authorities. Let $H(p)$ and $A(p)$ be the hub and authority score of page $p$ respectively. These scores are defined such that the following equations are satisfied for all pages $p:$

$$
\left\{\begin{array}{l}
H(p)=\Sigma_{u \in V \mid p \rightarrow u} A(u) \\
A(p)=\Sigma_{v \in V \mid v \rightarrow p} H(v)
\end{array}\right.
$$

where $H(p)$ and $A(p)$ are normalized for all web pages and calculated by the number of links. In our approach, we use the sum of authority and hub score as the score of a page, i.e., Score $(p)=H(p)+A(p)$, because pages with high authority scores are expected to have relevant content, whereas pages with high hub scores are expected to contain links to relevant content. However, for some high-scoring pages with few backward links but a large number of forward links, they are highly likely to be spam pages because they point to many irrelevant pages but only few pages refer to themselves. We use the number of backward links to measure similarity because our purpose is to find neighboring pages that are similar to the original page. According to [17], two web pages are similar in terms of prominence if they have similar amounts of backward links, which means there are similar amounts of web pages referring to them. Therefore, we modify above the equation as:

$$
\left\{\begin{array}{l}
H(p)=\Sigma_{u \in S \mid p \rightarrow u} \omega(p) \cdot A(u) \\
A(p)=\Sigma_{v \in S \mid v \rightarrow p} \omega(p) \cdot H(v)
\end{array}\right.
$$

where $\omega(p)$ is the weighting parameter calculated by the relevance value of a neighboring page to the original page. $\omega(p)$ is defined as the following:

$$
\omega(p)=\frac{1}{|\log N-\log N(p)|+1}
$$

where $N$ is the number of backward links of the original page, and $N(p)$ is the number of backward links of neighboring page $p$. In this way, if the number of backward links for page $p$ is similar to the original page, then a higher weight will be assigned to the page.

However, this formula does not represent the probability for a page to have a given number of backward links. For example, if an original page has 100 backward links, then the difficulty of finding a neighboring page with a similar amount of backward links is higher than that for an original page, which only has 5 backward links. Therefore, we extend Equation (5) as follows:

$$
\omega(p)=\frac{1}{|\log N-\log N(p)|+1} \cdot N
$$


which means if the original page has a higher amount of backward links, then its neighboring pages should have higher weights to better match the calculation for page score.

We then have the similarity score $\operatorname{Sim}(u, v)$ denoting the similarity between $u$ and $v$, which represents their importance against other pages and the similar recommendation they propagate to other pages in the graph. The graph is built from neighboring pages, which means most of the pages are from the same domain. Thus, $u$ and $v$ likely belong to the same web genre. For example, Michael Jordan's wiki page has numerous backward and forward links, most of which are NBA related, while its neighboring page, namely, Kobe Bryant's wiki page, has similar recommendations to other pages in the web graph according to the linking information. The similarity score is defined as below by adopting the Jaccard measure [10], which is commonly used in information retrieval to measure similarity:

$$
\operatorname{Sim}(u, v)=\frac{\sum_{i=1}^{n} \min \left(\operatorname{Score}\left(v_{i}, u\right), \operatorname{Score}\left(v_{i}, v\right)\right)}{\sum_{i=1}^{n} \max \left(\operatorname{Score}\left(v_{i}, u\right), \operatorname{Score}\left(v_{i}, v\right)\right)}
$$

where $u, v \in V$.

We then select the top $K$ neighboring pages with the highest similarity scores to extract information and construct features similar to the On-Page features we discussed in Section 3.1. We use these features from neighboring pages with On-Pages features to identify the genre of a web page. Details are given in the next section.

\subsection{Multiple Classifier Combination Module}

To achieve good performance in genre identification, we train multiple classifiers using the features acquired from the previous section and combine the classifiers to get a single prediction score. Most genre identification algorithms are based on machine learning techniques. Support Vector Machines(SVM) [28] is a powerful learning method based on Structural Risk Maximization theory, which aims to minimize the generalization error instead of relying on the empirical error on training data alone. In our approach, we have three classifiers, namely, SVM Contents, SVM On-Page Features, and SVM Neighboring Pages. SVM Contents is based on the textual information on the page as described in Section 3.1 while SVM On-Page Features adds structural information in the feature set. SVMs based on textual and structural information have been used by various researchers for genre identification, such as in [13,31,34], as discussed in Section 2. SVM Neighboring Pages is based on the textual and structural information in the selected neighboring pages. We further design a Multiple Classifier Combination (MCC) module to combine the three classi- 
fiers because the intuition is that the combination of homogeneous classifiers using heterogeneous features can improve the final result [29].

Assume each classifier based on SVM produces a unique decision as we define each web page $p$ as belonging only to one genre. We compare the results among all three classifiers, and the final output depends on the reliability of the decision confidences delivered by the participating classifiers. We apply the concept of Decision Template (DT) to avoid the case in which the classifiers make independent errors [20]. DT uses the outputs from all classifiers to calculate the final support, which is also called confidence score, based on a matrix for each class.

Assume each classifier produces the output $E_{i}(p)=\left[d_{i 1}(p), \ldots, d_{i|G|}(p)\right]$, where $d_{i j}(p)$ is the membership degree given by the classifier $E_{i}$ that a web page $p$ belongs to the genre $j, j=1, \ldots,|G|$. The outputs of all classifiers can be represented by a decision matrix $D P$ as follows:

$$
D P(p)=\left(\begin{array}{ccc}
d_{11}(p) & \ldots & d_{1|G|}(p) \\
d_{21}(p) & \ldots & d_{2|G|}(p) \\
d_{31}(p) & \ldots & d_{3|G|}(p) \\
\vdots & \\
d_{N 1}(p) & \ldots & d_{N|G|}(p)
\end{array}\right) .
$$

The membership degree $d_{i j}(p)$ is calculated using the training set $T_{j}, j \leq|G|$, where $T_{j}$ is the training set for each genre and $|G|$ is the number of genres.

$$
d_{i j}(p)=\frac{\operatorname{Ind}\left(T_{j}, i\right)}{|G|}
$$

where $\operatorname{Ind}\left(T_{j}, i\right)$ is an indicator function with value 1 only if the output from the classifier $E_{i}$ based on $T$ is the same as the output based on training set $T_{j}$, and the classifier assigns the page to genre $j$. Otherwise, we assign 0 to the function. At this stage, we have the membership degree for each page $p$ belonging to a genre $j$ and stored in a matrix $D P(p)$.

We then calculate the confidence score ConfidenceScore ${ }_{j}(p)$ of page $p$ using various rules from the $D P(p)$ for each genre $j$, and pick the genre with the highest confidence score. Assume $N$ is the number of classifiers, we apply minimum, maximum, and average rules for the matrix above to consider the diversity among multiple classifiers:

$$
\begin{aligned}
& \text { MinimumRules : ConfidencScore }{ }_{j}(p)=\operatorname{Min}_{i=1}^{N}\left(d_{i j}(p)\right) \\
& \text { MaximumRules : ConfidenceScore }{ }_{j}(p)=\operatorname{Max}_{i=1}^{N}\left(d_{i j}(p)\right) \\
& \text { AverageRules : ConfidenceScore }{ }_{j}(p)=\operatorname{Mid}_{i=1}^{N}\left(d_{i j}(p)\right)
\end{aligned}
$$




\section{Evaluations}

We discuss the evaluation results in this section. To evaluate our approach in depth, we not only evaluated the performance of individual classifiers and the MCC algorithm, but also compared them against existing work on different corpora.

\subsection{Corpora and Data Preparation}

In our experiment, we used the updated version from two popular corpora, KI04 and 7-Web collection [34]. We updated the web pages using a search engine so that we can get the latest contents and their neighboring pages. These corpora are composed of English web pages. Each web page is associated with a specific source URL address and belongs to a single genre class. KI-04 corpus (Table 1) is composed of 1205 HTML web pages, which are divided into eight genres, and 7-Web corpus (Table 1) contains 1400 HTML web pages from seven genres.

Table 1: KI-04 and 7-Web Dataset

\begin{tabular}{|c|c|c|c|}
\hline Genres (KI-04) & $\#$ & Genres (7-Web) & $\#$ \\
\hline ARTICLE & 127 & BLOG & 200 \\
\hline DOWNLOAD & 151 & E-SHOP & 200 \\
\hline DISCUSSION & 205 & FAQs & 200 \\
\hline PORTRAYAL-PRIVATE & 126 & ONLINE NEWSPAPER FRONTPAGE & 200 \\
\hline PORTRAYAL-NON PRIVATE & 163 & PERSONAL HOME PAGE & 200 \\
\hline LINK COLLECTION & 127 & LISTING & 200 \\
\hline HELP & 139 & SEARCH PAGE & 200 \\
\hline SHOP & 167 & & \\
\hline
\end{tabular}

To evaluate if our approach can perform well in case the web pages do not have much textual information, we also manually and randomly collected 2000 such pages with four genres from the Internet called IV-12 dataset as shown in Table 2. The four genres are movie homepages, e.g., Transformer $4^{2}$, photography websites, e.g., Dreams Time ${ }^{3}$, video sharing websites, e.g., YouTube $^{4}$ and music websites, e.g., Music.com ${ }^{5}$.

We crawled neighboring pages for the web pages in all three corpora by using the "<a href $>$ " tag for forward links, and AHREFS", which is a online tool to analyze websites, for backward links. The latest crawled was done in November 2012 with total 372325 neighboring pages after removed those broken links. We pre-processed all web pages including neighboring pages before

\footnotetext{
2 http://www.transformersmovie.com/

3 http://www.dreamstime.com/

4 http://www.youtube.com

5 http://www.music.com

6 http://ahrefs.com/
} 
Table 2: IV-12 Dataset

\begin{tabular}{|c|c|}
\hline Genres & $\#$ \\
\hline Movie Homepages & 500 \\
\hline Photography Websites & 500 \\
\hline Video Sharing Websites & 500 \\
\hline Music Download Websites & 500 \\
\hline
\end{tabular}

using classifiers to train. We selected and tokenized text into words, as well as remove numbers, non-letter characters, common stop words ${ }^{7}$, and special characters. We then selected stem terms using the Lovins stemmer [25] and calculated the TF-IDF value for each remaining term for each page. All above steps were implemented by using Word Vector Tool ${ }^{8}$ and htmlparser ${ }^{9}$.

\subsection{Evaluation Results}

We evaluated our approach based on metrics adopted by some previous works $[21,30,36]$.

Pairwise F1 is defined as the harmonic mean of pairwise precision and pairwise recall. Pairwise precision is the number of true positives divided by the total number of elements labeled as belonging to the positive class. Recall in this context is defined as the number of true positives divided by the total number of elements that actually belong to the positive class. They are defined as Precision $=\frac{T P}{T P+F P}$, Recall $=\frac{T P}{T P+F N}$, and $F 1=\frac{2 \cdot \text { Precision } \cdot \text { Recall }}{\text { Precision }+ \text { Recall }}$.

$T P$ stands for True Positive, the number of items correctly labeled as belonging to the positive class; FP stands for False Positive, the items incorrectly labeled as belonging to the class; and $F N$ stands for False Negative, items that are not labeled as belonging to the positive class but should have been.

Since our data is multi-class, the evaluation is done in two binary classifications, one-class-against-the-rest and pairwise [3]. We used Macro-averaging [36] to measure the classification accuracy of both methods. Hence, precision and recall are first evaluated "locally" for each class, and then "globally" by averaging over the results of the different classes. Then, the Macro-averaging of precision Precision $_{M}$ and recall Recall $_{M}$ is represented as Precision $_{M}=$ $\frac{\sum_{i=1}^{|C|} \text { Precision }_{i}}{|C|}$, Recall $_{M}=\frac{\sum_{i=1}^{|C|} \text { Recall }_{i}}{|C|}$, where $|C|$ is the total number of classes.

\subsubsection{Evaluations of Neighboring Pages Selection}

We first evaluated our neighboring pages selection model by applying different similarity measures in KI-04, 7-Web, and IV-12 corpora. We selected the top $K$ neighboring pages with the highest similarity scores generated by different

\footnotetext{
7 http://www.textfixer.com/resources/common-english-words.txt

8 http://ostatic.com/wvtool

9 http://htmlparser.sourceforge.net/
} 
similarity measures and manually checked the pages to obtain the selection accuracy. The selection accuracy is measured using $\frac{M}{K}$, where $M$ is the number of neighboring pages with the same genre as the original page. GenreSim achieves better results than other models in all three corpora as shown in Fig. 5, and it performs particularly well on the IV-12 corpus which consists of web pages without sufficient textual information in both cases when $K=10$ and $K=20$. The performance drops for all similarity measures with the increase of $K$, but the margin of drop for GenreSim is smaller than that of others. Given that the performance is better when $K=10$, we decided to use only the top 10 neighboring pages to identify the genre of web pages.

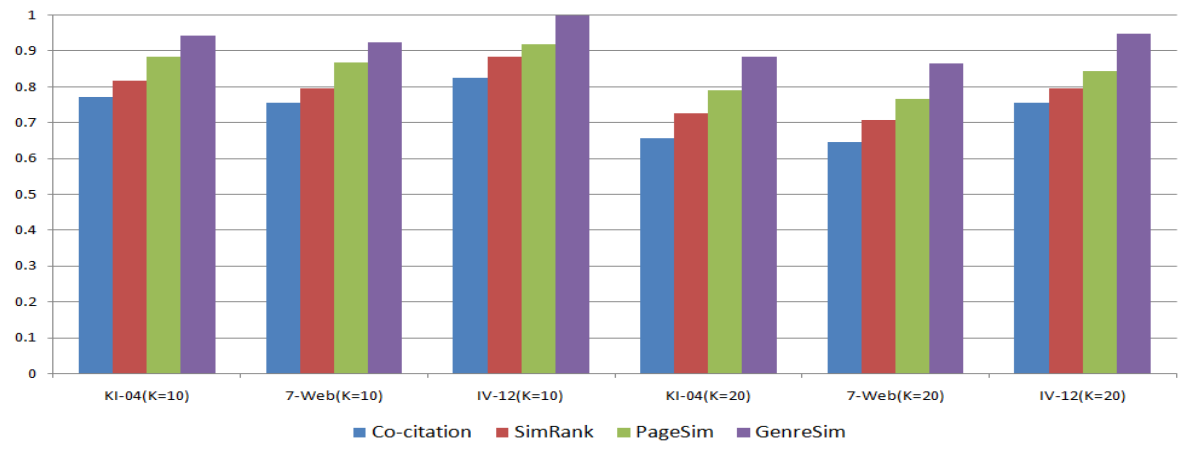

Fig. 5: Performance of Different Similarity Measures for Neighboring Pages Selection

\subsubsection{Comparisons of Individual Classifiers}

This section discusses the comparisons of individual classifiers. As discussed earlier, we implemented SVM Contents and SVM On-Page Features classifiers, which represent existing works, to compare with our approach based on neighboring pages, namely, SVM Neighboring Pages classifier. In addition, to evaluate the performance of our model in selecting the most relevant neighboring pages for genre identification, we also generated another classifier with neighboring pages by using the Google Similar Pages function ${ }^{10}$ to return the first 10 neighboring pages. All classifiers were implemented by Weka API 11, and we used RBFKernel [40] for SVM with default Weka' settings. In the learning phase, we adopt the 10-fold cross validation to split the dataset for classifier training and testing.

Fig. 6 shows the Macro-averaging of four classifiers for the pages in the test datasets KI-04, 7-Web, and IV-12 for pairwise classification, while Fig. 7 shows the Macro-averaging of four classifiers for the pages in the test datasets KI-04, 7-Web, and IV-12 for one-class-against-the-rest classification. As seen

10 http://www.google.com/help/features.html

11 http://www.cs.waikato.ac.nz/ml/weka/ 
in the results in both tables, no significant difference is observed between SVM Contents classifier and SVM On-Page Features classifier on KI-04 and 7-Web datasets because the dimension of textual information feature is much higher than that of the structural information feature, and the On-Page features are dominated by textual feature. The classifier based on the feature of neighboring pages generated from our algorithm outperforms other classifiers, except in the 7-Web dataset in the case of pairwise classification, where SVM Contents classifier obtains better precision result. This result is attributed to the 7Web dataset consisting of web pages with rich textual/structural information, but with relatively insufficient or irrelevant textual/structural information in neighboring pages. In the case of IV-12, the dataset containing web pages without much textual information, the SVM Contents and SVM On-Page Features achieve better results than other two datasets. However, all the three corpora are different from one another and should be treated individually because they are in different sizes and different types of web pages. The key finding in this test case is the SVM Neighboring Pages classifier performs much better than the other classifiers, around 15\% improvement from traditional On-Page method. Its superiority is beyond the cases on the other two corpora. This finding emphasizes our conclusion that the information in neighboring pages can significantly improve the genre identification performance when the textual information is limited and insufficient. In addition, the classifier based on the neighboring pages generated by our model outperforms the one generated by Google Similar Pages function in all cases, which also supports the favorable results of our neighboring pages selection approach.

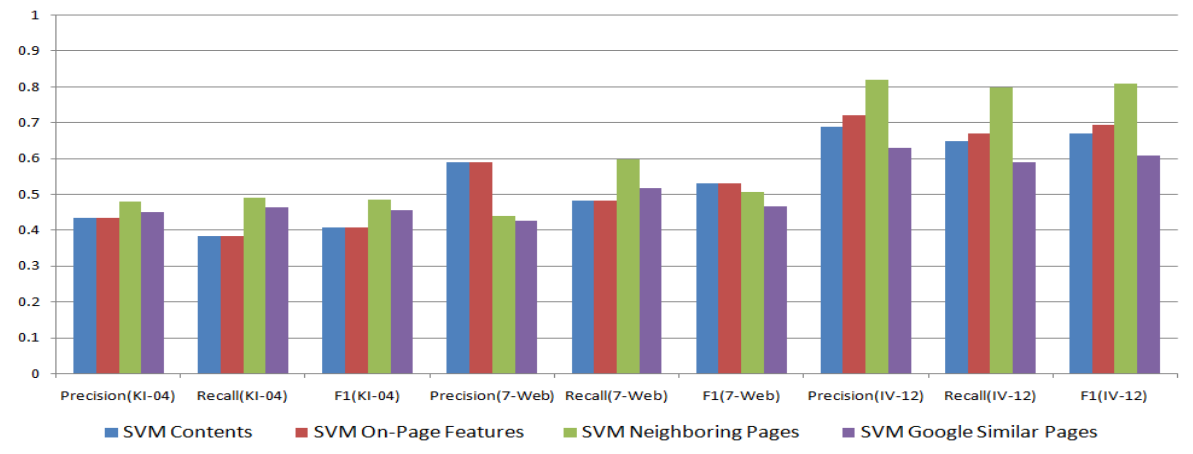

Fig. 6: Macro-averaging of Individual Classifiers in Pairwise Classification

\subsubsection{Performance of $M C C$}

We evaluated MCC in both Pairwise Classification and One-Class-Against-theRest Classification. Fig. 8 and 9 show the Macro-averaging results of MCC by applying different rules to calculate the confidence score as described in Section 3. As shown in the tables, the maximum rule produces better results than 


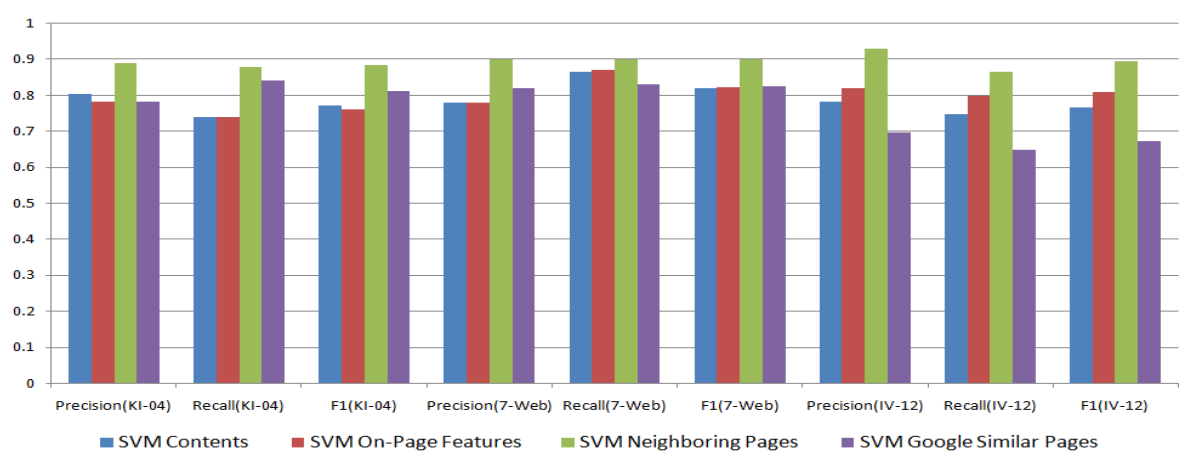

Fig. 7: Macro-averaging of Individual Classifiers in One-Class-Against-the-Rest Classification

the other two rules in all three data corpora. From the results, we discover that, by integrating all the information we can access, the identification results can be evidently increased. Especially for the 7-Web dataset, the result of MCC outperforms that by SVM Contents and SVM On-Page Features, which indicating that the information extracted from neighboring pages can effectively enrich the knowledge about the target web pages, and improve the final identification results.

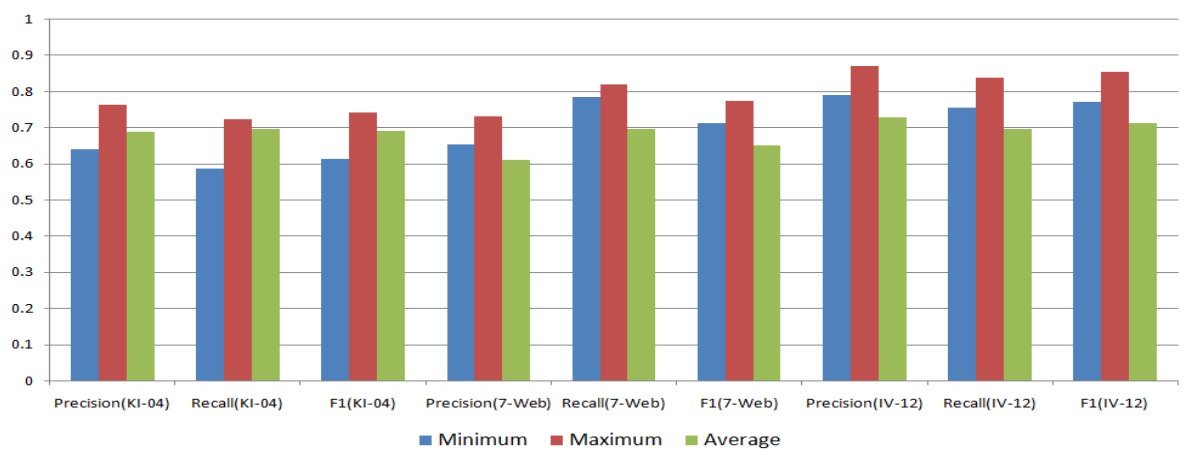

Fig. 8: Macro-averaging of MCC in Pairwise Classification

\subsubsection{MCC vs. Individual Classifiers}

We also compared the results between MCC and individual classifiers, as shown in Fig. 10 and 11. We selected the results of maximum rule in MCC and the best results from three features produced by SVM to compare. The results show that MCC improves around $20 \%$ in KI-04 and 7-Web in Pairwise Classification and outperforms SVM in One-Class-Against-the-Rest Classification 


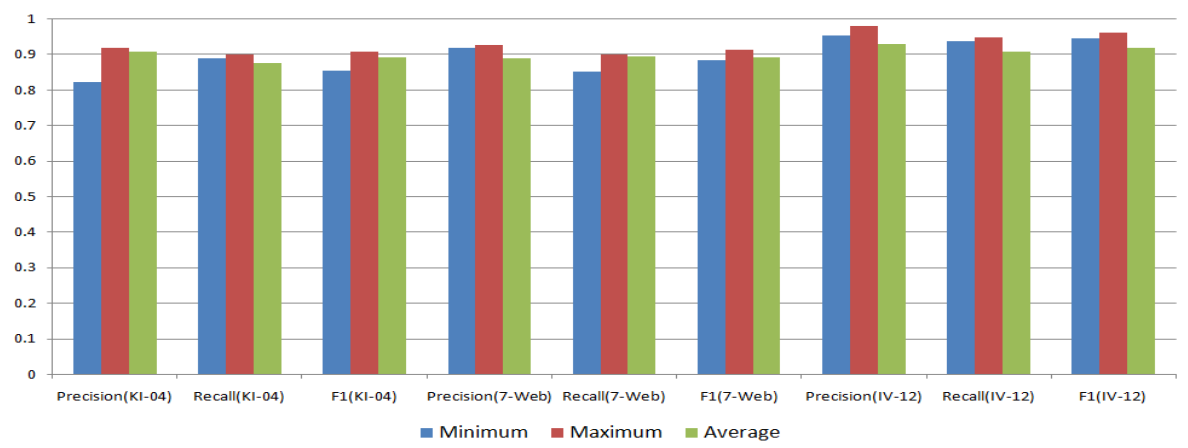

Fig. 9: Macro-averaging of MCC in One-Class-Against-the-Rest Classification

at an average of $4 \%$, which means MCC can improve considerably in the case of multi-class classification.

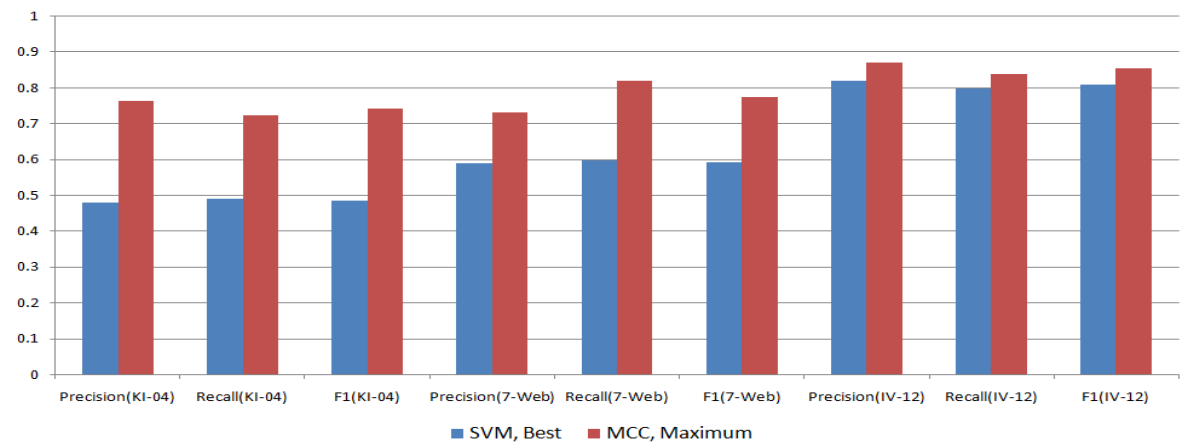

Fig. 10: MCC vs. Individual Classifiers in Pairwise Classification

\subsubsection{MCC vs. Other Ensemble Methods}

To evaluate the performance of MCC, in addition to using the common majority voting (MV) [22] algorithm as the baseline method for comparison, we also implemented the Random Feature Subspacing Ensemble (RFSE) algorith$\mathrm{m}$ based on the latest work [31] without considering the case of an unknown genre to suit our purpose. We used the same features in all three methods. With respect to the parameters of RFSE, we applied the standard setting to the value of $k 1$ and $k 2$, which are 10 and 100 respectively. Fig. 12 and 13 show that all three methods achieve better results than the individual classifiers. The performance of MCC is just slightly better than that of MV and RFSE except in the IV-12 dataset, which showed an approximate $3 \%$ average improvement. This result indicates that our proposed method is suitable for those web pages without much textual and structural information. 


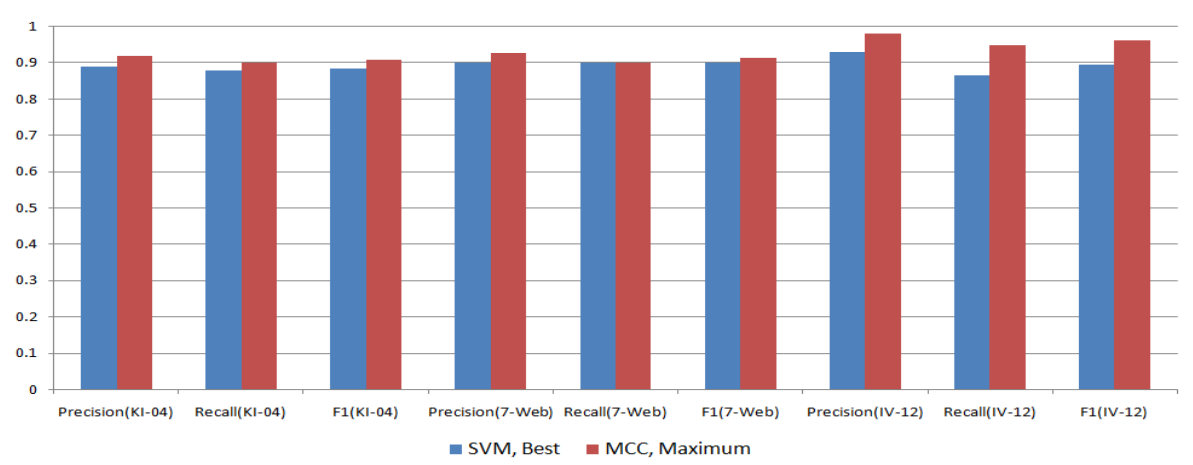

Fig. 11: MCC vs. Individual Classifiers in One-Class-Against-the-Rest Classification

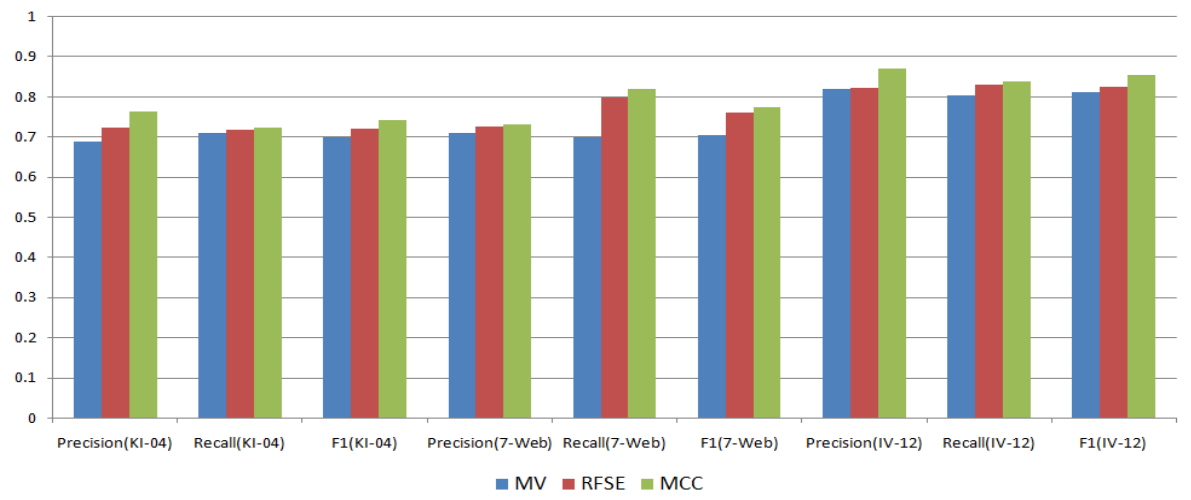

Fig. 12: MCC vs. Other Ensemble Methods in Pairwise Classification

\subsubsection{Significance Tests}

We also designed two significance tests to compare the performance of two systems, one is the s-test designed for micro-level analysis [41], the other is the standard t-test ${ }^{12}$ designed for macro-level analysis.

For the s-test, we used the following notations:

- $N$ is the number of decisions by each system;

- $n$ is the number of times that the decision by two systems are different;

- $k$ is the number of times that the decision by one system is correct and the decision of the other system is incorrect.

The P-value (1-sided) is computed using the binomial distribution:

12 http://en.wikipedia.org/wiki/T-test 


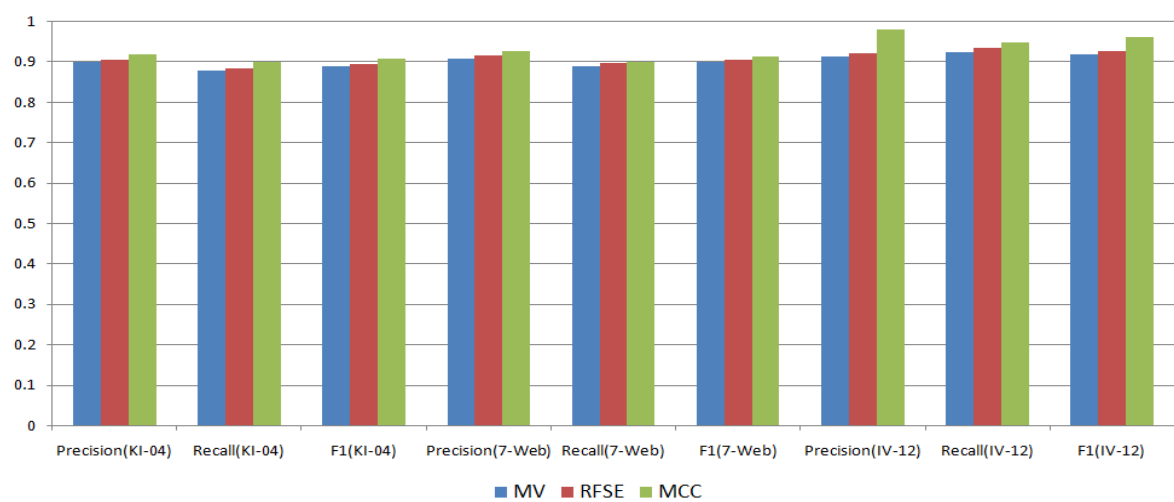

Fig. 13: MCC vs. Other Ensemble Methods in One-Class-Against-the-Rest Classification

$$
P(Z)=\left\{\begin{array}{l}
\sum_{i=k}^{n}\left(\begin{array}{c}
n \\
i
\end{array}\right) \times 0.5^{n}, \quad Z \geq k \\
\sum_{i=0}^{k}\left(\begin{array}{c}
n \\
i
\end{array}\right) \times 0.5^{n}, \quad Z<k
\end{array}\right.
$$

where $Z=\frac{k-0.5 n}{0.5 \sqrt{n}}$.

Tables 3, 4 and 5 summarize the results of statistical significance tests on the $F 1$ score on three corpora where " $>$ " or " $\gg$ " indicates a better classifier. We only perform significance tests on pairwise classification because its $F 1$ value is not as apparent as in the case of one-class-against-the-rest classification among classifiers. Not surprisingly, MCC dominates both micro and macro level significance tests. It is not only better than the other two ensemble methods, but also significantly better than individual classifiers.

With regard to the individual classifiers, we cannot say SVM Neighboring Pages is significantly better than others on the KI-04 and 7-Web corpora as their outcomes on s-test and t-test are not in agreement. However, from the results in Table 5, we find that SVM Neighboring Pages outperforms al1 individual classifiers, proving our method can improve the web page genre identification performance when the textual information of web pages is limited. 
Table 3: Statistical significance test results on KI-04

\begin{tabular}{|c|c|c|c|}
\hline sysA & sysB & s-test & t-test \\
\hline MCC & RFSE & $>$ & $>$ \\
\hline MCC & MV & $>$ & $>$ \\
\hline MCC & SVM Neighboring Pages & $\gg$ & $\gg$ \\
\hline SVM Neighboring Pages & SVM On-Page Features & $>$ & $>$ \\
\hline SVM Neighboring Pages & SVM Contents & $>$ & $>$ \\
\hline SVM Neighboring Pages & SVM Google Similar Pages & $\sim$ & $>$ \\
\hline SVM On-Page Features & SVM Contents & $\sim$ & $\sim$ \\
\hline SVM Contents & SVM Google Similar Pages & $<$ & $<$ \\
\hline
\end{tabular}

$\gg$ or $\ll$ means $\mathrm{P}$-value $\leq 0.01$;

$>$ or $<$ means $0.01<$ P-value $<0.05$;

$\sim$ means $\mathrm{P}$-value $>0.05$.

Table 4: Statistical significance test results on 7 -Web

\begin{tabular}{|c|c|c|c|}
\hline sysA & sysB & s-test & t-test \\
\hline MCC & RFSE & $>$ & $>$ \\
\hline MCC & MV & $>$ & $>$ \\
\hline MCC & SVM Neighboring Pages & $\gg$ & $\gg$ \\
\hline SVM Neighboring Pages & SVM On-Page Features & $\sim$ & $<$ \\
\hline SVM Neighboring Pages & SVM Contents & $\sim$ & $<$ \\
\hline SVM Neighboring Pages & SVM Google Similar Pages & $\sim$ & $>$ \\
\hline SVM On-Page Features & SVM Contents & $\sim$ & $\sim$ \\
\hline SVM Contents & SVM Google Similar Pages & $>$ & $>$ \\
\hline
\end{tabular}

$\gg$ or $\ll$ means $\mathrm{P}$-value $\leq 0.01$;

$>$ or $<$ means $0.01<\mathrm{P}$-value $<0.05$;

$\sim$ means $\mathrm{P}$-value $>0.05$.

Table 5: Statistical significance test results on IV-12

\begin{tabular}{|c|c|c|c|}
\hline sysA & sysB & s-test & t-test \\
\hline MCC & RFSE & $>$ & $>$ \\
\hline MCC & MV & $>$ & $>$ \\
\hline MCC & SVM Neighboring Pages & $>$ & $>$ \\
\hline SVM Neighboring Pages & SVM On-Page Features & $>$ & $>$ \\
\hline SVM Neighboring Pages & SVM Contents & $>$ & $>$ \\
\hline SVM Neighboring Pages & SVM Google Similar Pages & $\gg$ & $\gg$ \\
\hline SVM On-Page Features & SVM Contents & $\sim$ & $\sim$ \\
\hline SVM Contents & SVM Google Similar Pages & $>$ & $>$ \\
\hline
\end{tabular}

$\gg$ or $\ll$ means $\mathrm{P}$-value $\leq 0.01$;

$>$ or $<$ means $0.01<\mathrm{P}$-value $<0.05$;

$\sim$ means $\mathrm{P}$-value $>0.05$. 


\subsubsection{Comparisons using Full Text}

To make our work more completed, we have also compared the SVM Neighboring Pages classifier to one of representative existing works [37] using full text information of web pages. We used 4-grams in this experiment, which is the best feature the authors found. The details are shown in Fig. 14 and 15. From the results, we can see that both methods' performance are in the same level on KI-04 and 7-Web corpora but SVM Neighboring Pages is better on IV-12. For KI-04 and 7-Web corpora, we notice that the original web pages already contain sufficient textual feature, so the SVM 4-grams algorithm can work well. However, our SVM Neighboring Pages classifier can still achieve similar performance by applying information from neighboring pages. This result can evidently support the effect of the selected neighboring pages. Furthermore, it is not surprising that for the IV-12 corpus consisting of web pages with limited textual information, our approach can outperform the one using only textual features on original pages, and this benefits from the auxiliary information from neighboring pages.

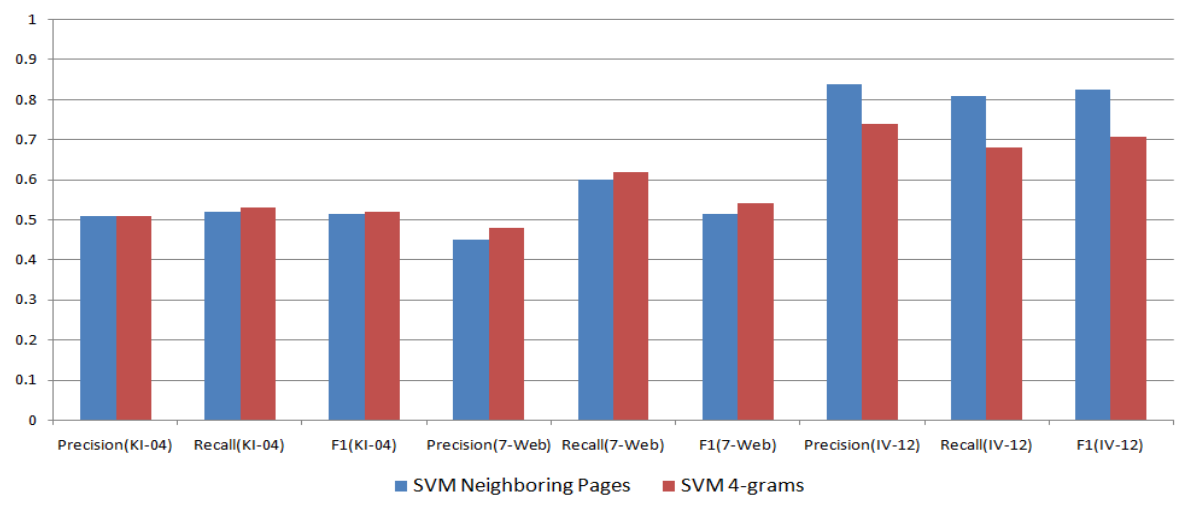

Fig. 14: SVM Neighboring Pages vs. SVM 4-grams in Pairwise Classification

\subsubsection{Error Analysis}

Last but not least, we analyzed the errors for the SVM Neighboring Pages classifier and the MCC method, and categorized the errors as shown in Table 6 and 7 . The figure showed in the tables is the average percentage of errors from pairwise classification and one-class-against-the-rest classification. In definition, "error" here means the classifier or method has not classified the web page to the right class. In Table 6 and 7, "Only Classifier" or "Only Method" means the errors are only made by the SVM Neighboring Pages classifier or MCC but not any others. "Less Information" means the neighboring pages 


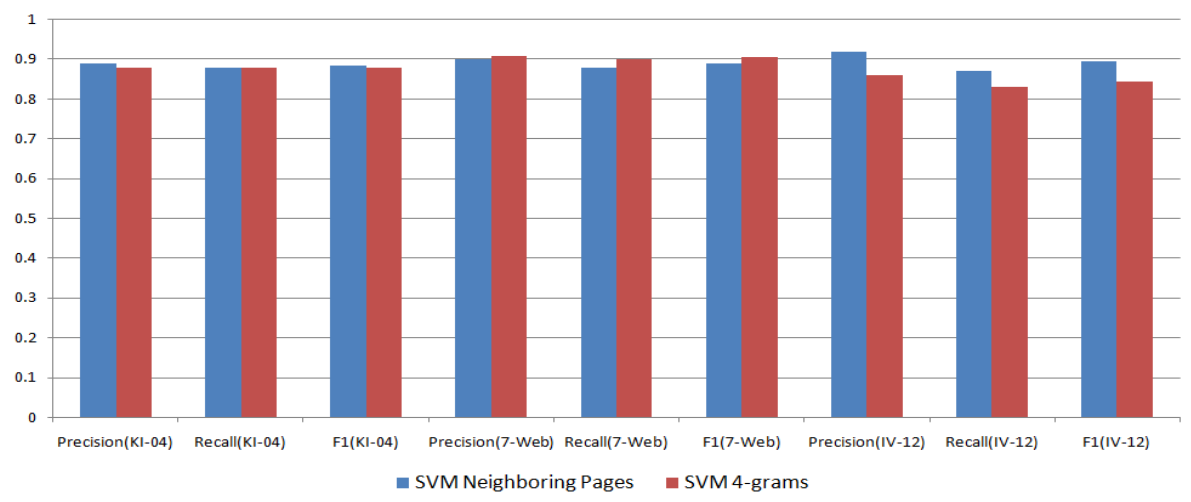

Fig. 15: SVM Neighboring Pages vs. SVM 4-grams in One-Class-Against-the-Rest Classification

have less information than the original page to be classified. "All Classifiers" or "All Methods" means the errors are made by all approaches.

From the Table 6, we know the SVM Neighboring Pages classifier heavily relies on the information in the selected pages. For example, in the 7-Web dataset, $19.4 \%$ errors made by the SVM Neighboring Pages classifier are not happened by other classifiers. In other words, if we use information from the original page, these mistakes can be avoided. The reason of the classifier made these mistakes mainly because there is no sufficient useful information in selected neighboring page, e.g., we found that $78.5 \%$ errors in 7 -Web dataset, the neighboring pages have less information than the original page. For instance, there is no any backward and forward links for the "PHP_025.htm", then obviously we can not get any information from neighboring pages. Of course, none of classifiers can identify this kind of page because there is also no much textual or structural information in the page. Another error example is "PHP_028.htm", though we can get a few its neighboring pages but most of linked pages are irrelevant to it. We also found a pattern that the errors often happened in personal homepage, which is one of genres in 7-Web dataset. According to our observations, personal homepages usually contain few external links or the linked pages are belong to different genres, which is the limitation using the information in neighboring pages to identify.

According to the results in Table 7, since MCC and other two methods are combined by all classifiers, there is no obvious patterns in all three corpora. In addition, the percentage of errors with less information is around $50 \%$, which means the cause of errors does not depend on the amount of information we have. 
Table 6: Error Statistics of SVM Neighboring Pages classifier

\begin{tabular}{|c|c|c|c|}
\hline Errors & KI-04 & $7-$ Web & IV-12 \\
\hline Only Classifier & $8.5 \%$ & $19.4 \%$ & $2.9 \%$ \\
\hline Less Information & $62.6 \%$ & $78.5 \%$ & $64 \%$ \\
\hline All Classifiers & $43.5 \%$ & $54.4 \%$ & $62.1 \%$ \\
\hline
\end{tabular}

Table 7: Error Statistics of MCC

\begin{tabular}{|c|c|c|c|}
\hline Errors & KI-04 & $7-$ Web & IV-12 \\
\hline Only Method & $3.5 \%$ & $3.4 \%$ & $3.8 \%$ \\
\hline Less Information & $46.5 \%$ & $48.5 \%$ & $52 \%$ \\
\hline All Methods & $88.5 \%$ & $85.4 \%$ & $82.2 \%$ \\
\hline
\end{tabular}

\section{Conclusions and Future Work}

In this paper, we addressed the challenge in the problem of web page genre identification, which significantly benefits the improvement of web search. We focused on the situation that the web pages only contain limited textual information, in which the conventional approaches based on On-Page features only may not correctly identify the genre of these pages due to the insufficient information available. To tackle this problem, the idea of making use of neighboring web pages was proposed to potentially enrich the useful feature information. For a web page to be identified, among its neighboring pages, there can be several ones of the similar genre as the original page. Therefore, we are able to select those pages and collect the useful textual information from them, which can help to identify the genre of the engaged web page. Such mechanism is especially effective when the textual information of the engaged web page is insufficient. Based on these considerations, we designed our framework to select appropriate neighboring pages and effectively identify the engaged web page's genre. To filter out the most appropriate neighboring pages, GenreSim model was designed according to the graph-based similarity measure. To make best use of the neighboring pages, we designed a multiple classifier combination mechanism to integrate the information in neighboring pages with the On-Page features. Such framework successfully improved the web page genre identification performance, especially for the cases in which the textual information is limited for the web pages to be identified. The advantages of our proposed framework were proved and supported by extensive experimental evaluation on different well known corpora with error analysis. Conclusively, the proposed framework is an essential and valuable complement for the research of web page genre identification, and provides effective alternative solution for dealing with the extreme situations, i.e., insufficien$\mathrm{t}$ textual information provided. In the future, we will focus on investigating dynamic weighting for individual classifier to further improve the ensemble performance of multiple classifiers. 


\section{References}

1. M. Abramson and D. W. Aha. Whats in a url? genre classification from urls. Intelligent Techniques for Web Personalization and Recommender Systems AAAI Technical Report, pages 1-8, 2012.

2. A. Arasu, J. Cho, H. Garcia-Molina, A. Paepcke, and S. Raghavan. Searching the web. ACM Transactions on Internet Technology, pages 2-43, 2001.

3. S. Bernhard, J. C. Burges, and A. J. Smola. Advances in kernel methods: support vector learning. MIT Press.

4. L. Bjroneborn. Genre connectivity and genre drift in a web of genres. Genres on the Web:Computational Models and Empirical Studies, pages 255-274, 2011.

5. E. Boese and A. Howe. Effects of web document evolution on genre classification. Proc. of the ACM 14th Conference on Information and Knowledge Management, pages 632 $639,2005$.

6. G. Chen and B.Choi. Web page genre classification. Proc. of 2008 ACM symposium on Applied computing, pages 2353-2357, 2008.

7. L. Dong, C. Watters, J. Duffy, and M. Shepherd. An examination of genre attributes for web page classification. Proc. of the 41th Annual Hawaii International Conference on System Sciences, pages 129-138, 2008.

8. S. M. Eissen and B. Stein. Genre classification of web pages: User study and feasibility analysis. In Biundo S., Fruhwirth T. and Palm G. (eds.). KI, pages 256-269, 2004.

9. A. Finn and N. Kushmerick. Learning to classify documents according to genre. Journal of the American Society for Information Science and Technology, 7:257-262, 2006.

10. A. K. Jain and R. C. Dubes. Algorithms for clustering data. Prentice-Hall, 1988.

11. C. Jebari. A New Centroid-based Approach for Genre Categorization of Web Pages. JLCL, 24(1):73-96, 2009.

12. G. Jeh and J. Widom. Simrank: a measure of structural-context similarity. Proc. of the 8th ACM SIGKDD international conference on Knowledge discovery and data mining, pages $538-543,2002$.

13. I. Kanaris and E. Stamatatos. Web page genre identification using variable-length character n-grams. 19th IEEE International Conference on Tools with Artificial Intelligence, $7(1): 3-10,2007$

14. A. Kennedy and M. Shepherd. Automatic identification of home pages on the web. Proc. of the 38th Annual Hawaii International Conference on System Sciences, pages 99-108, 2005.

15. B. Kessler, G. Nunberg, and H. Shutze. Automatic detection of text genre. Proc. of the 35th Annual Meeting of the Association for Computational Linguistics, pages 32-38, 1997.

16. Y. Kim and S. Ross. Formulating representative features with respect to genre classification. In Genres on the Web, Computational Models and Empirical Studies, 42:129-147, 2011.

17. J. M. Kleinberg. Hubs, authorities, and communities. ACM Comput. Surv., 31(4es), 1999.

18. J. M. Kleinbery. Authoritative sources in a hyperlinked environment. Journal of the $A C M, 46(5): 604-632,1999$.

19. K. P. Kumari and A. Reddy. Performance improvement of web page genre classification. International Journal of Computer Applications, 53(10):24-27, September 2012. Published by Foundation of Computer Science, New York, USA.

20. L. I. Kuncheva, J. C. Bezdek, and R. P. Duin. Decision templates for multiple classifier fusion. Pattern Recognition, 34(2):299-314, 2001.

21. A. H. F. Laender, M. A. Goncalves, R. G. Cota, A. A. Ferreira, R. L. T. Santos, and A. J. C. Silva. Keeping a digital library clean: New solutions to old problems. Proc. of the 8th ACM Symposium on Document Engineering, pages 257-262, 2008.

22. L. Lam and C. Y. Suen. Majority vote of even and odd experts in a polychotomous choice situation. Theory and Decision, pages 13-36, 1996.

23. Y. Lee and S. Myaeng. Text genre classification with genre-revealing and subjectrevealing features. Proc. of the 25th ACM SIGIR Conf. on Research and Development in Information Retrieval, pages 145-150, 2002. 
24. Z. Lin, I. King, and M. R. Ly. Pagesim: A novel link-based similarity measure for the world wide web. Proc. of the 5th International Conference on Web Intelligence, pages 687-693, 2006.

25. J. Lovins. Development of a stemming algorithm. Mechanical Translation and Computational Linguistics 11, pages 22-31, 1968

26. J. E. Mason, M. Shepherd, J. Duffy, V. Keselj, and C. Watters. An n-gram based approach to multi-labeled web page genre classification. 46th Hawaii International Conference on System Sciences, 0:1-10, 2010.

27. A. Mehler, R. Gleim, and A. Wegner. Structural uncertainty of hypertext types. an empirical study. Proceedings of the International Workshop:Towards Genre-Enabled Search Engines: The Impact of NLP, pages 13-19, 2007.

28. T. Mitchell. Machine learning. McGraw-Hill, 1997.

29. C. Orrite, M. Rodriguez, F. Martinez, and M. Fairhurst. Classifier ensemble generation for the majority vote rule. Progress in Pattern Recognition, Image Analysis and Applications, pages 340-347, 2008.

30. D. A. Pereira, B. N. Ribeiro, N. Ziviani, H. F. Alberto, A. M. Goncalves, and A. A. Ferreira. Using web information for author name disambiguation. Proc. of the 9th ACM/IEEE-CS joint conference on Digital libraries, pages 49-58, 2009.

31. D. Pritsos and E. Stamatatos. Open-set classification for automated genre identification. Advances in Information Retrieval, pages 207-217, 2013.

32. X. Qi and B. Davison. Classifiers without borders: Incorporating fielded text from neighboring web pages. Proc. of the 31st Annual International ACM SIGIR Conference on Research and Development on Information Retrieval, pages 643-650, 2008.

33. G. Salton and M. J. McGill. Introduction to modern information retrieval. 1986.

34. M. Santini. Automatic genre identification: Towards a flexible classification scheme. BCS IRSG Symposium: Future Directions in Information Access, 2007.

35. M. Santini. Characterizing genres of web pages: Genre hybridism and individualization. Proceedings of the 40th Annual Hawaii International Conference on System Sciences, 2007.

36. F. Sebastiani. Machine learning in automated text categorization. ACM Computing Surveys (CSUR), 34(1):1-47, 2002.

37. S. Sharoff, Z. Wu, and K. Markert. The web library of babel: evaluating genre collections. LREC-2010, pages 3063-3070, 2010.

38. E. Stamatatos, N. Fakotakis, and G. Kokkinakis. Text genre detection using common word frequencies. Proc. of the 18th Int. Conf. on Computational Linguistics, pages 808-814, 2000.

39. B. Stein, E. Zu, and M. Sven. Is web genre identification feasible? Proc. of the 2006 conference on ECAI 2006, pages 815-816, 2006.

40. V. Vapnik. The nature of statistical learning. Springer-Verlag, 1995

41. Y. Yang and X. Liu. A re-examination of text categorization methods. SIGIR, pages 42-49, 1999.

42. J. Zhu, X. Zhou, and G. Fung. Enhance web pages genre identification using neighboring pages. Web Information System Engineering, pages 282-289, 2011. 